20

\title{
Исследование сыворотки крови у крыс с трансплантированной холангиокарциномой с использованием спектроскопии комбинационного рассеяния света
}

\author{
(C) А.А. Манькова ${ }^{1}$, О.П. Черкасова 2,3, Ф, Е.Н. Лазарева ${ }^{4,5}$, А.Б. Бучарская 6 , П.А. Дьяченко ${ }^{4,5}$, \\ Ю.В. Кистенев ${ }^{4,7}$, Д.А. Вражнов ${ }^{4,8}$, В.Е. Скиба ${ }^{4}$, В.В. Тучин ${ }^{4,5,9}$, А.П. Шкуринов ${ }^{1,3}$ \\ ${ }^{1}$ Московский государственный университет имени М.В.Ломоносова, \\ 119991 Москва, Россия \\ ${ }^{2}$ Институт лазерной фризики Сибирского отделения РАН, \\ 630090 Новосибирск, Россия \\ ${ }^{3}$ Институт проблем лазерных и информационных технологий РАН — фрилиал ФНИЦ „Кристаллография и фротоника“ РАН, \\ 140700 Шатура, Россия \\ ${ }^{4}$ Национальный исследовательский Томский государственный университет, \\ 634050 Томск, Россия \\ ${ }^{5}$ Саратовский национальный исследовательский государственный университет имени Н.Г. Чернышевского, \\ 410012 Саратов, Россия \\ ${ }^{6}$ Саратовский государственный медицинский университет им. В.И. Разумовского, \\ 410012 Саратов, Россия \\ ${ }^{7}$ Сибирский государственный медицинский университет, \\ 634050 Томск, Россия, \\ ${ }^{8}$ Институт фризики прочности и материаловедения Сибирского отделения РАН, \\ 634055 Томск, Россия \\ ${ }^{9}$ Институт проблем точной механики и управления РАН, \\ 410028 Саратов, Россия \\ "e-mail: o.p.cherkasova@gmail.com
}

Поступила в редакцию 10.01.2020 г.

В окончательной редакции 31.01.2020 г.

Принята к публикации 28.02.2020 г.

Образцы сыворотки крови здоровых крыс и крыс с привитыми опухолями через 14 и 28 суток после трансплантации клеток холангиокарциномы исследованы с применением метода спектроскопии комбинационного рассеяния света (КРС). Показано, что интенсивность полосы амид I отличается в спектрах сыворотки крови в зависимости от стадии развития опухоли и коррелирует с концентрацией белка в образцах. У части животных наблюдается достоверное увеличение концентрации глюкозы в крови, приводящее к гликированию белков и изменению формы полосы амид І. Появление полосы на $1670 \mathrm{~cm}^{-1}$ наиболее ярко выражено в спектрах образцов на 28 сутки эксперимента и может быть связано с увеличением содержания $\beta$ структурных элементов в конформации белка. Использование метода главных компонент позволяет оценить различия спектров КРС в целом в сыворотке крови групп контрольных крыс и крыс на 28-е сутки после трансплантации опухоли.

Ключевые слова: комбинационное рассеяние света, сыворотка крови, экспериментальный рак печени, метод главных компонент.

DOI: $10.21883 / O S .2020 .07 .49568 .73-20$

\section{Введение}

Исследование интегральных показателей, характеризующих различия между биологическими образцами, полученными от здоровых и больных людей, имеет большое значение, так как диагностика, основанная на одном маркере, часто неинформативна [1,2]. Спектроскопия комбинационного рассеяния света (КРС) является мощным аналитическим методом, который можно применять в дифференциальной диагностике заболеваний [3]. Спектр КРС представляет собой молекулярный отпечаток образца и дает количественную информацию о его химическом составе [4]. Биохимические изменения в клетках и тканях, связанные с различными заболеваниями, могут привести к значительным изменениям в спектрах КРС [3-5]. Применение спектроскопии КРС для дифференциальной диагностики рака мозга, молочной железы, кожи и ряда других видов онкологии описано в работе [6]. Анализ крови может обеспечить минимально инвазивную диагностику для многих заболеваний, включая онкологические [7]. Так, было показано, что спектроскопия КРС в сочетании с анализом главных компонент и линейным дискриминационным анализом позволила провести различие между образцами сыворотки крови 
больных раком молочной железы и здоровых добровольцев [8], между образцами пациентов с гепатоцеллюлярной карциномой и без нее [9], выявлять молекулярные маркеры различных подтипов глиобластомы $[10,11]$.

Спектры КРС представляют собой данные высокой размерности, поэтому применение классических методов статистического анализа для проверки гипотезы о разделимости исследуемых групп по различиям в их спектрах КРС затруднительно. Использование метода главных компонент снижает размерность данных, выявляет информативные участки спектров и дает возможность визуализировать результаты, что позволяет оценить возможность создания модели данных для дифференцирования представителей различных экспериментальных групп [3,8,9,12-15].

Холангиокарцинома (альвеолярный рак печени) представляет собой злокачественную опухоль, которая образуется из мутированных эпителиальных клеток желчных протоков. Заболеваемость этим типом рака значительно возросла в последние годы [16].

Целью работы является изучение спектров КРС сыворотки крови крыс в динамике развития экспериментальной холангиокарциномы с применением метода главных компонент для анализа данных.

\section{Материалы и методы}

\section{Характеристика животных}

В работе моделировали развитие альвеолярного рака печени (холангиокарцинома, РС1), осуществляя введение $0.5 \mathrm{ml} 25 \%$ опухолевой взвеси в растворе Хэнкса подкожно в область лопатки белым беспородным крысам-самцам [17]. Животных выводили из эксперимента на 14-й и 28-й день после трансплантации опухоли [18]. Все эксперименты на животных выполняли в соответствии с международными этическими нормами [19]. На основании данных результатов биохимического анализа животные были разделены на 2 основные группы: группа 1 - животные с нормальным уровнем глюкозы; группа 2 - животные с повышенным уровнем глюкозы. Каждая из основных групп была поделена на 3 подгруппы: подгруппы 1.1 и 2.1 - здоровые животные контрольных групп; подгруппы 1.2 и 2.2 - животные через 14 дней после трансплантации опухоли; подгруппы 1.3. и 2.3 - животные через 28 дней после трансплантации опухоли. Содержание белка и глюкозы в сыворотке крови определяли стандартными методами [18]. Для обработки полученных данных биохимического анализа был использован пакет прикладных статистических программ SSPS-13.0. Результаты представляли в виде медианы и квартилей значений (табл. 1). Показатель достоверности различий $(p)$ данных между группами определяли с использованием критерия Манна-Уитни.

Содержание белка и глюкозы в сыворотке крови экспериментальных животных приведены в табл. 1.

\section{Спектроскопия KP}

В работе использовали КР-микроскоп DXR Raman Microscope (Thermo Scientific) с увеличением 10x (цифровая апертура 0.25), длина волны возбуждения $780 \mathrm{~nm}$ (группа 1) и $532 \mathrm{~nm}$ (группа 2), диапазон $80-3200 \mathrm{~cm}^{-1}$. Измерения спектров КРС проводили на образцах сыворотки крови. Каждый образец представлял собой каплю объемом 10-16 $\mu$, нанесенную на специальную алюминиевую подложку, в которой были вырезаны одинаковые лунки в форме воронки диаметром 5 и глубиной $2 \mathrm{~mm}$ (рис. 1). Такая форма подложки приводит к равномерному распределению образца при высыхании. При работе с одинаковыми объемами наносимой жидкости можно считать, что распределение разных проб в каждой лунке и толщина пленки образца после высыхания будут одинаковыми.

Измерения проводили после полного высыхания образца, которое проходило в течение $5 \mathrm{~min}$ при комнатной температуре. Регистрацию спектра КРС проводили в трех точках в центре лунки и усредняли. Каждый спектр усредняли по 1000 сканам для улучшения соотношения сигнал/шум, время накопления составило $16 \mathrm{~min}$. Экспериментальные спектры КРС были обработаны с использованием метода сравнения [20] и метода катящегося колеса [21].

\section{Методы анализа спектральных данных}

Для сравнительного анализа спектров КРС сыворотки крови крыс в динамике развития экспериментального рака печени была изучена возможность применения альтернативных методов коррекции фона по сравнению с классическим подходом. Одним из использованных методов для вычитания фона была функция „msbackadj“ из пакета программного обеспечения Matlab R2018®. Данный метод состоит из трех этапов. Сначала производится оценка линии фона на основе нескольких, сдвинутых друг относительно друга окон. Шаг сдвига составлял 200 условных единиц, что соответствовало сдвигу частоты в спектре на такую же величину. Ширина окна также была выбрана в 200 условных единиц. Данные величины подбиралась таким образом, чтобы максимально уменьшить влияние фона. Далее линия фона аппроксимируется в точках заданного окна, используя кусочно-гладкую кубическую интерполяцию, сохраняющую форму кривой. На последнем этапе фон корректируется с учетом пиков сигнала.

Также было использовано вычитание фона на основе алгоритма (arPLS), представленного в работе [22] и на основе алгоритма (als) из работы [23]. Данные алгоритмы реализованы в библиотеке Rampy (версии 0.4.4 Stable) [24] на языке Python (версии 3.6.8).

После того как коррекция линии фона была завершена, спектральные данные на частотах ниже 80 и выше $2580 \mathrm{~cm}^{-1}$ не были приняты к рассмотрению изза низкого отношения сигнал-шум. Дальнейший анализ 
Таблица 1. Содержание белка и глюкозы в сыворотке крови крыс

\begin{tabular}{l|c|c|c|c|c|c}
\hline \multicolumn{1}{c|}{ Группы } & \multicolumn{3}{|c|}{ Группа 1} & \multicolumn{3}{c}{ Группа 2 } \\
\hline Параметры & $\begin{array}{c}1.1 \text { контроль } \\
n=8\end{array}$ & $\begin{array}{c}1.214 \text { дней } \\
n=4\end{array}$ & $\begin{array}{c}1.328 \text { дней } \\
n=6\end{array}$ & $\begin{array}{c}2.1 \text { контроль } \\
n=6\end{array}$ & $\begin{array}{c}2.214 \text { дней } \\
n=4\end{array}$ & $\begin{array}{c}2.328 \text { дней } \\
n=5\end{array}$ \\
\hline Общий белок, g/1 & $83[67 ; 86.3]$ & $73[69.5 ; 77.3]$ & $57^{* *, \#}[55 ; 64]$ & $76.5[60.7 ; 85.5]$ & $65[60.2 ; 77.2]$ & $63^{*}[61 ; 73.5]$ \\
\hline Альбумин, g/1 & $37[35.5 ; 39.3]$ & $39[36.5 ; 40.8]$ & $21^{* *, \#[19 ; 23]}$ & $38[32 ; 39.7]$ & $34[30.5 ; 38.2]$ & $31^{*}[27.5 ; 33.5]$ \\
\hline Глюкоза, mol/1 & $5.2[4.9 ; 5.7]$ & $7.8^{*}[6.7 ; 8.9]$ & $5.4[5 ; 5.7]$ & $6.0[4.9 ; 9.5]$ & $14.1^{* *}[12.4 ; 17.2]$ & $9.1^{*, \#}[7.6 ; 11.9]$
\end{tabular}

Примечание. $n$ - количество животных; данные представлены как медиана и квартили биохимических показателей; достоверность отличий: * $p \leq 0.05$ по сравнению с контрольными подгруппами 1.1 или 2.1 в соответствующей группе; ** $p \leq 0.01$ по сравнению с контрольными подгруппами 1.1 или 2.1 в соответствующей группе; ${ }^{*} p \leq 0.01$ по сравнению с подгруппами 1.2 или 2.2 в соответствующей группе.

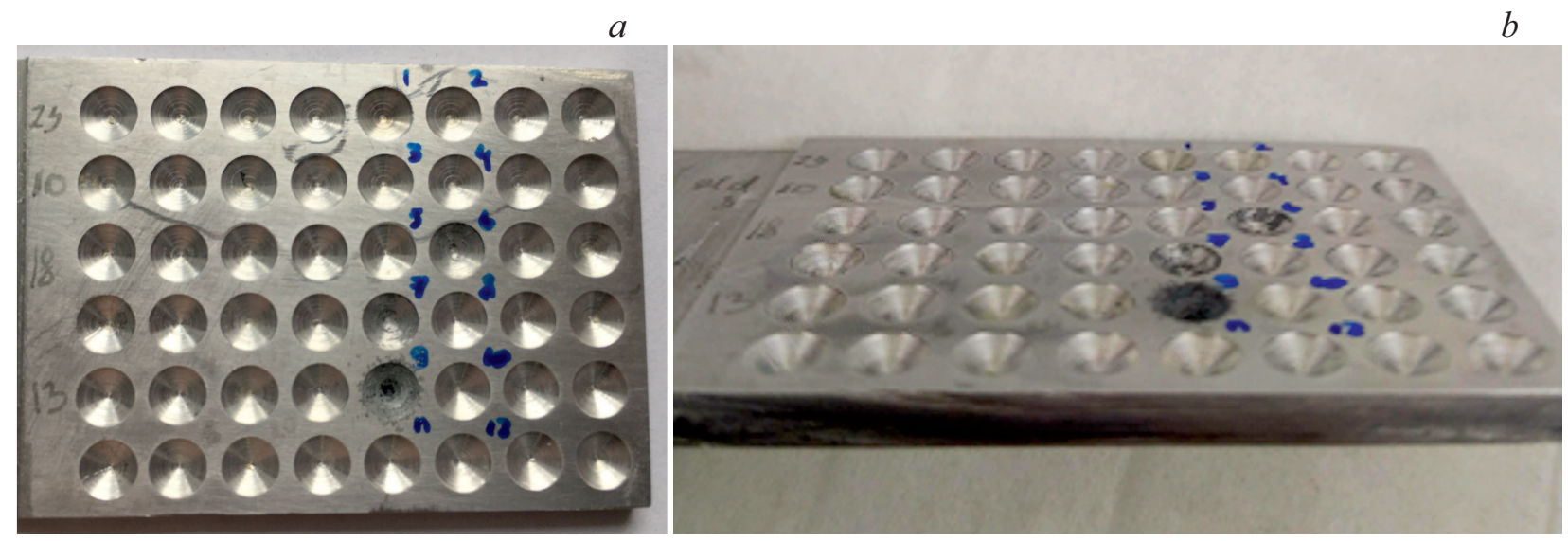

Рис. 1. Специальная алюминиевая подложка: $a-$ вид сверху; $b-$ вид сбоку.

был выполнен с помощью метода главных компонент (МГК).

\section{Результаты и обсуждение}

Сыворотка крови содержит почти 90\% воды, около 6.6-8.5\% белков и других органических и минеральных соединений, которые являются промежуточными или конечными продуктами обмена веществ, переносимых кровью [25]. Различные компоненты крови оказывают значительное влияние на спектры КРС, причем их вклад может изменяться при развитии патологических состояний $[26,27]$. В диапазоне $500-1800 \mathrm{~cm}^{-1}$ находится большое количество линий, индивидуальных для каждого органического соединения $[28,29]$. Это в основном область валентных и деформационных колебаний. По наличию или отсутствию конкретных линий в спектре в этом диапазоне можно определить как само исследуемое вещество, так и его концентрацию в биологическом образце $[28,30]$.

В группе 1 в динамике развития опухоли наблюдается статистически значимое уменьшение концентрации как общего белка, так и альбумина в сыворотке крови (см. табл. 1). Содержание альбумина в сыворотке крови крыс подгруппы 1.3 (28 дней после трансплантации опухоли)
Таблица 2. Основные типы колебаний в молекуле альбумина

\begin{tabular}{l|l}
\hline $1230-1320 \mathrm{~cm}^{-1}$ & $\begin{array}{l}\text { С-N валентные } \\
\text { и N-Н изгибные колебания (Амид III) }\end{array}$ \\
\hline $1480-1580 \mathrm{~cm}^{-1}$ & $\begin{array}{l}\text { С-N валентные } \\
\text { и N-Н изгибные колебания (Амид II) }\end{array}$ \\
\hline $1600-1690 \mathrm{~cm}^{-1}$ & $\begin{array}{l}\text { Валентные C=O } \\
\text { колебания (Амид I) }\end{array}$
\end{tabular}

почти в два раза меньше, чем в крови здоровых крыс и крыс подгруппы 1.2 (14 дней после трансплантации опухоли). Альбумин составляет бо́льшую часть белков сыворотки крови [25] и его содержание изменяется в динамике развития экспериментального рака печени [18], поэтому особенное внимание в спектрах КРС обратили на линии, характерные для спектра альбумина, а именно линии колебаний пептидных связей амид I, II и III, а также линий ряда аминокислотных остатков [29,31]. Типы основных колебаний в молекуле альбумина приведены в табл. 2.

На рис. 2 приведены спектры КРС для группы 1, регистрируемые при длине волны возбуждения $780 \mathrm{~nm}$. Показан диапазон $1500-1750 \mathrm{~cm}^{-1}$. Обработка спектров 


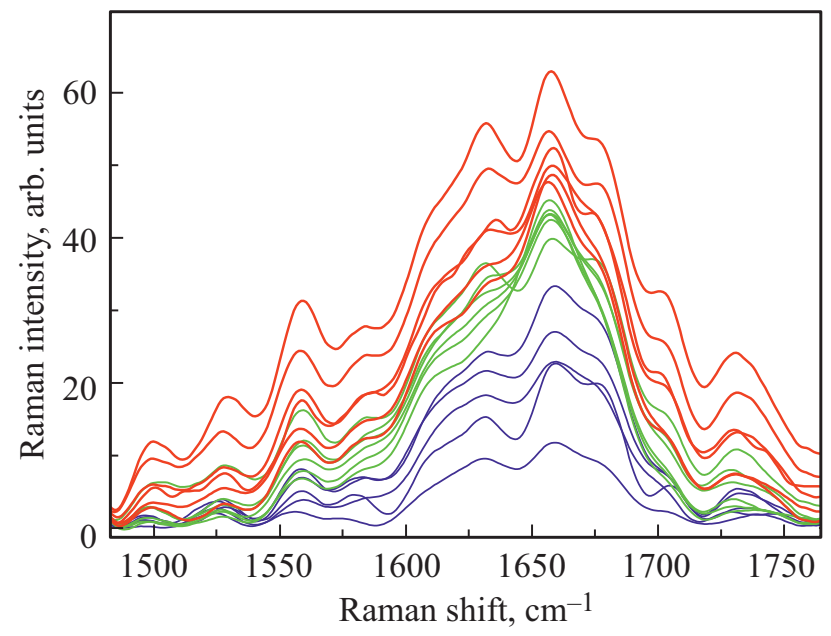

Pис. 2. Спектры КР в диапазоне $1500-1700 \mathrm{~cm}^{-1}$ для образцов группы 1, длина волны возбуждения $780 \mathrm{~nm}$ : подгруппа 1.1 - красная линия, подгруппа 1.2 - зеленая линия, подгруппа 1.3 - синяя линия.

проведена методом катящегося колеса [21]. Наиболее интенсивные линии наблюдаются на частотах 1558, 1631, 1657 и $1677 \mathrm{~cm}^{-1}$. Данные линии наблюдаются во всех образцах. Положение линий не изменяется в спектрах КРС сыворотки крови разных экспериментальных групп, но их амплитуда отличается: она тем меньше, чем меньше содержание белка в образце. Наименьшая амплитуда линий наблюдается для образцов группы 1.3 (синие линии на рис. 2). Наблюдается хорошая корреляция между содержанием белка в образцах и интенсивностью указанных линий.

У животных группы 2 в процессе развития опухоли концентрация белка в сыворотке крови также уменьшается, но в меньшей степени, чем в группе 1. Однако уровень глюкозы в крови на 14 сутки достоверно выше как относительно уровня животных контрольных групп 1 и 2, так и относительно животных с таким же сроком опухоли группы 1 (подгруппа 1.2, табл. 1). При дальнейшем росте опухоли уровень глюкозы в группе 2 уменьшается, но остается достоверно выше нормальных значений и значений в сыворотки крови у животных группы 1. Известно, что высокий уровень глюкозы в крови приводит к гликированию белков крови и изменению их структуры $[32,33]$. Показателем изменения структуры альбумина, как наиболее распространенного белка крови, является изменение структуры полосы амид I. Анализ линии амид I был проведен более детально. Для этого провели вычитание фона в диапазоне $1500-1700 \mathrm{~cm}^{-1}$ при помощи метода катящегося колеса [21]. Размер колеса, прокатываемого под графиком - 50 (рис. 3 ).

Как видно из рис. 3, наиболее интенсивные линии наблюдаются на частотах 1550, 1585, 1604 и $1656-1670 \mathrm{~cm}^{-1}$. Заметно изменение формы полосы амид I. Практически у всех образцов появляется полоса на частоте $1670 \mathrm{~cm}^{-1}$, что может свидетельствовать об увеличении содержания $\beta$-структурных элементов в конформации белка [34-43]. Как известно, альбумин имеет преимущественно $\alpha$-спиральные элементы $(67 \%)$ в своей структуре [44]. Возможно, увеличение $\beta$-структурных элементов связано с взаимодействием белка с другими компонентами сыворотки крови крыс в динамике развития опухоли. Наиболее интенсивной эта полоса становится в спектрах образцов подгруппы 2.3, в которых опухоль развивалась 28 суток (синие линии на рис. 3). Эта подгруппа имеет ряд различий в биохимическом составе крови по сравнению с животными контрольной группы (табл. 1), в частности характеризуется более высоким уровнем глюкозы, что может привести к гликированию белков, изменению их структуры, и находит свое отражения в спектрах КРС [44,45].

Для сравнения спектров КРС сыворотки крови крыс в динамике развития экспериментального рака печени была изучена возможность применения МГК для анализа данных. Следует отметить, что данный метод применяется для разведывательного анализа данных КРС, когда количество образцов мало [46,47]. Перед применением МГК необходимо было провести подготовку первичных экспериментальных данных, в частности корректировку фона. Как отмечалось выше, для сравнения методов выделения фона нами было использовано несколько различных подходов: функция „msbackadj“, вычитание фона на основе алгоритма (arPLS) [22] и на основе алгоритма (als) [23]. Лучший результат, согласно визуальной оценке (рис. 4), дает алгоритм arPLS, предложенный в работе [22]. На рис. 4 показан пример обработки КРС спектров для группы 2.

Заключительным этапом предварительной обработки спектральных данных служила векторная нормализация [48].

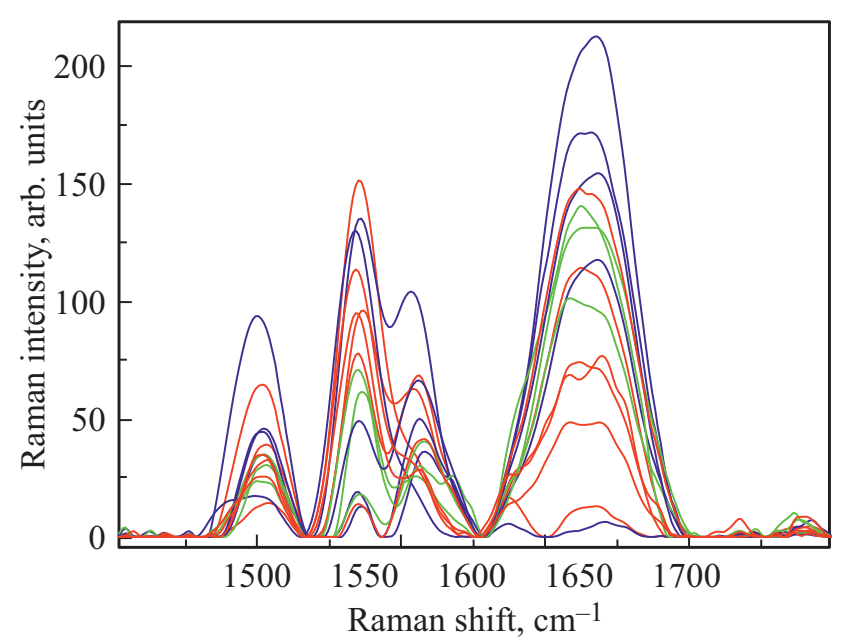

Рис. 3. Спектры КР в диапазоне $1500-1700 \mathrm{~cm}^{-1}$ для образцов группы 2, длина волны возбуждения $532 \mathrm{~nm}$ : подгруппа 2.1 - красная линия, подгруппа 2.2 - зеленая линия, подгруппа $2.3-$ синяя линия. 


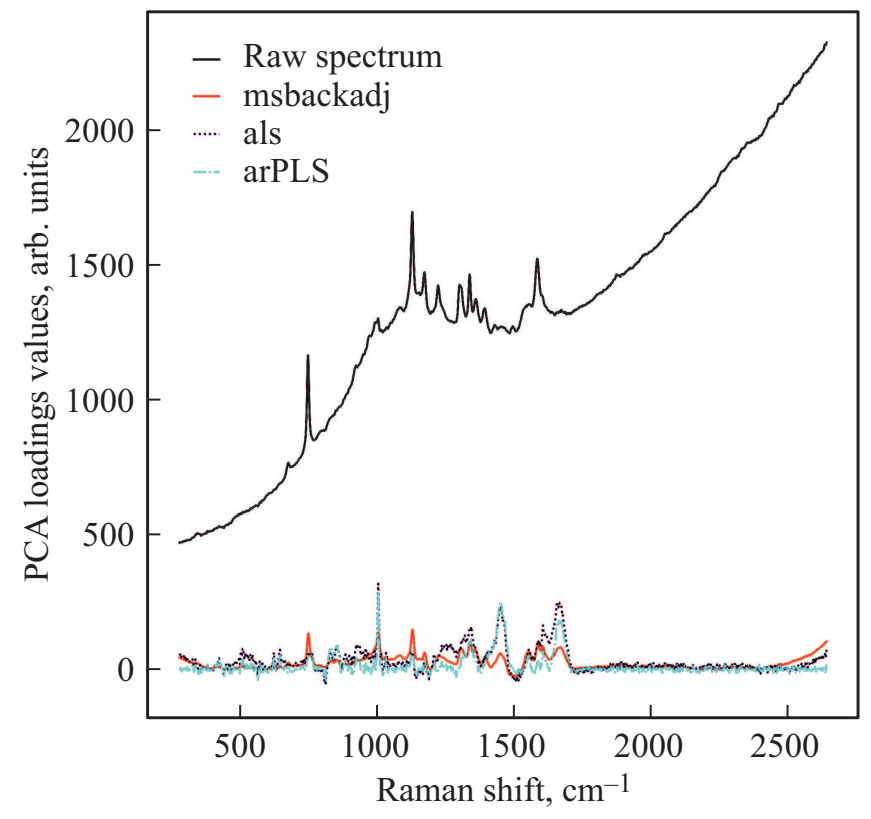

Pис. 4. Применение методов msbackadj, als, arPLS для вычитания фона спектров КРС группы 2.

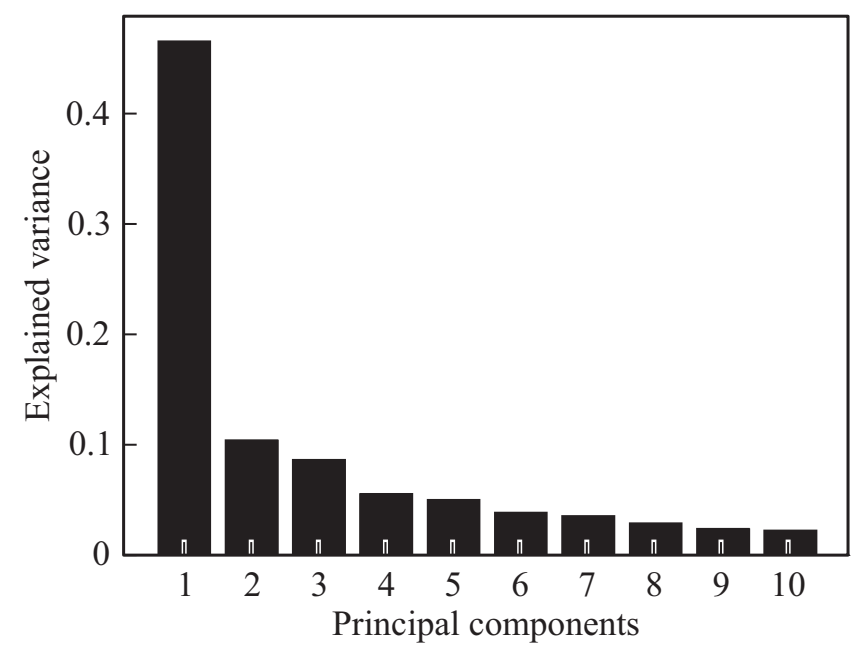

Рис. 5. Объясненная дисперсия главных компонент для группы 1.

Дальнейший анализ результатов проводили МГК с целью уменьшения размерности данных КРС, удаления шумов и визуализации расположения данных в редуцированном пространстве признаков. Следует отметить, что анализ матрицы нагрузок в методе МГК позволяет выделить наиболее значимые признаки [46]. Количество главных компонент определяет степень редукции пространства признаков и выбирается путем анализа объясненной дисперсией для исследуемого набора данных, например методом „сломанной трости“ (графики объясненной дисперсии представлены на рис. 5 и 6 для групп 1 и 2 соответственно).
Как видно на рис. 5, для группы 1 наиболее информативными и значимыми являются первая и вторая компоненты, которые составляют 97.8\% дисперсии.

Из рис. 6 видно, что для группы 2 наиболее информативными и значимыми являются первая, вторая и третья компоненты, составляющие 94.4\% дисперсии, что свидетельствует о наличии дополнительных факторов, оказывающих существенное влияние на структуру данных. Таким фактором может быть высокий уровень глюкозы в сыворотке крови, приводящий к образованию продуктов гликрования и гликированию белков, что может давать вклад в изменение спектральных данных по сравнению с группой 1.

На рис. 7 и 8 представлены распределения матрицы нагрузок для первой, второй и третьей главных компонент для групп 1 и 2 соответственно. Значительные по амплитуде пики свидетельствуют о важном вкладе соответствующих частот в дифференциацию групп, и

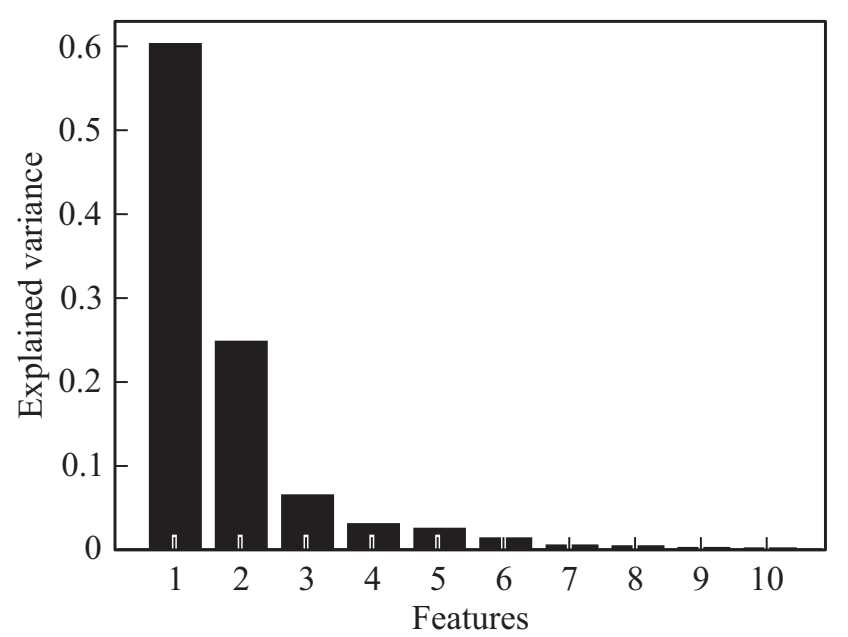

Рис. 6. Объясненная дисперсия главных компонент для группы 2.

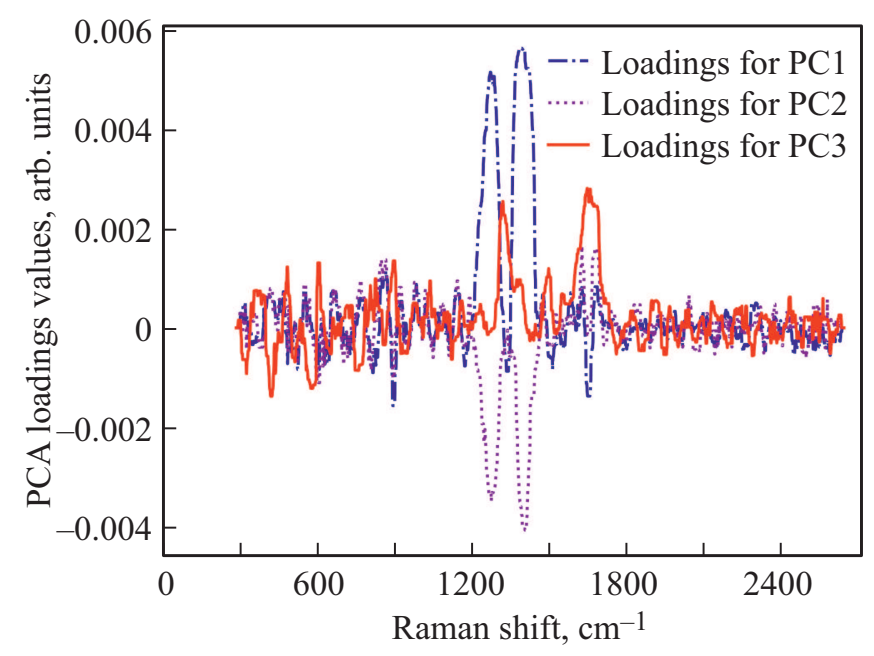

Рис. 7. Распределение матрицы нагрузок для 1-й, 2-й и 3-й главной компоненты для группы 1. 
их координаты согласуются с указанными выше результатами.

После уточнения количества главных компонент, содержащих бо́льшую долю информации, были построены возможные комбинации проекций двух главных компонент на плоскость. Выборочные результаты представлены на рис. 9 и 10. Метками в виде круга обозначены данные для групп крыс через 14 суток после трансплантации опухоли (подгруппы 1.2 и 2.2), треугольником через 28 суток после трансплантации опухоли (подгруппы 1.3 и 2.3), квадратом - для группы здоровых крыс (подгруппы 1.1 и 2.1).

Из рис. 9 видно, что в проекции на вторую главную компоненту подгруппы 1.1 и 1.2 образуют плотную группу, также наблюдается хорошее разделение данных подгруппы 1.3 от подгрупп 1.1 и 1.2 по второй главной компоненте. Заметим, что подгруппа 1.3 представляет собой разреженный кластер. Подгруппа 1.3 имеет достоверно низкий уровень как общего белка, так и альбумина

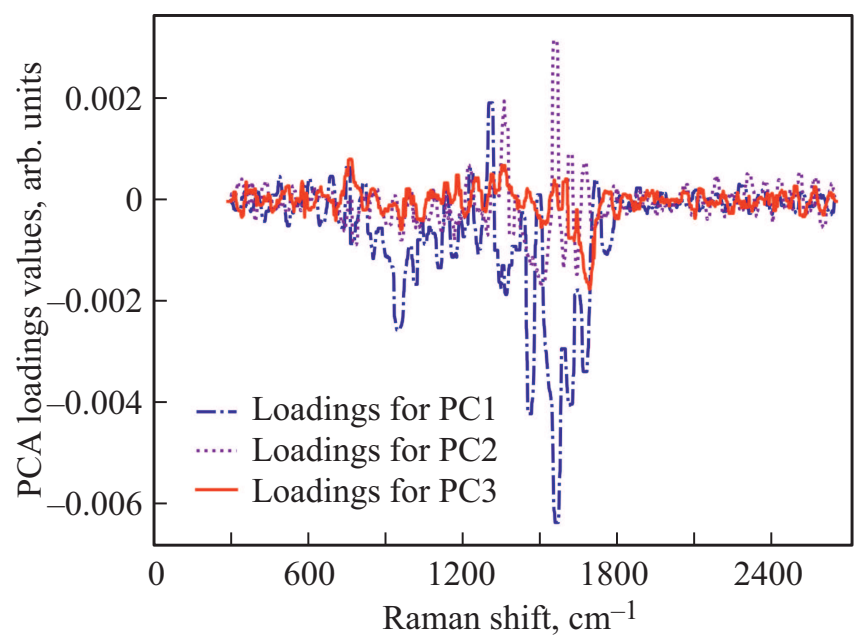

Pис. 8. Распределение матрицы нагрузок для 1-й, 2-й и 3-й главных компонент для группы 2.

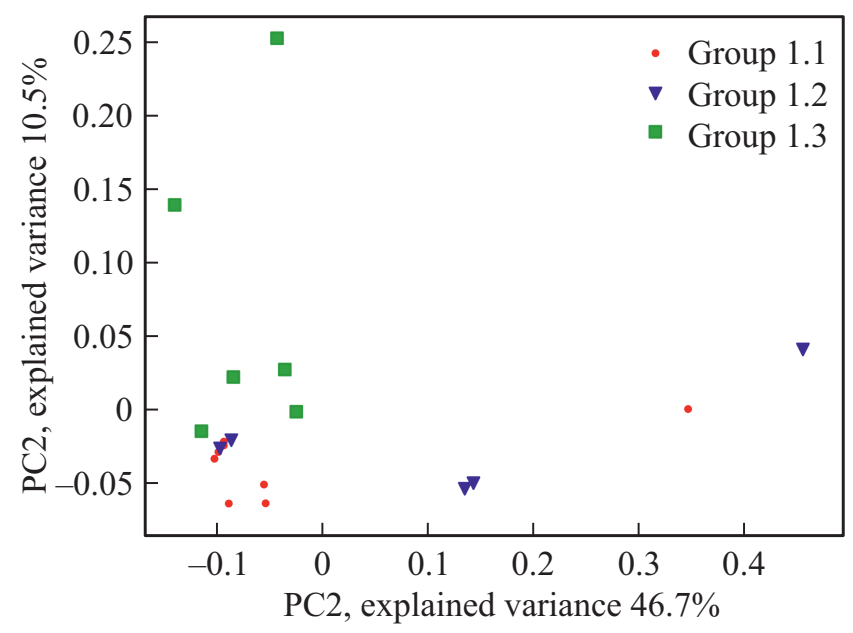

Рис. 9. Проекция 1-й и 2-й главных компонент на плоскость для группы 1.

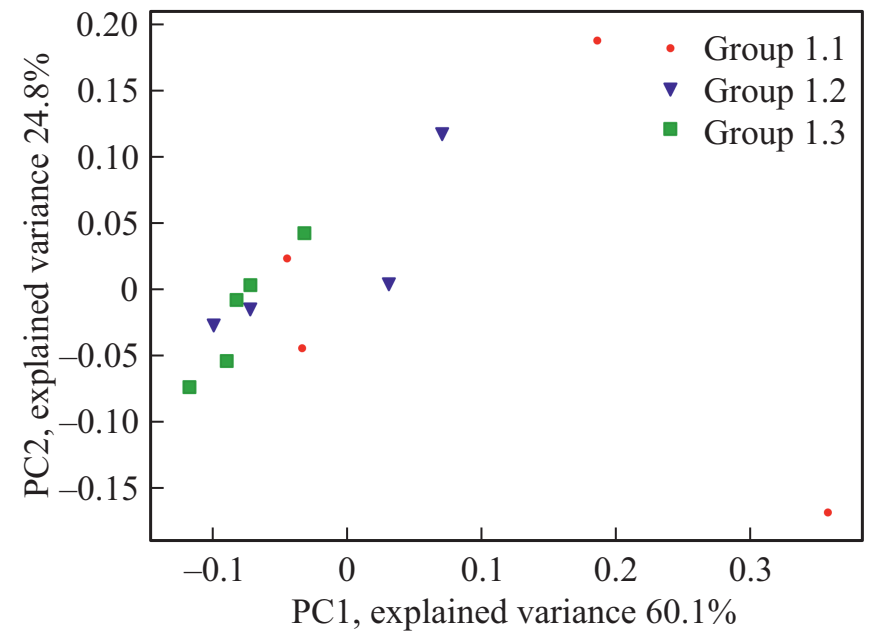

Рис. 10. Проекция 1-й и 2-й главных компонент на плоскость для группы 2.

в сыворотке крови по сравнению с подгруппами 1.1 и 1.2. В то же время наблюдается пересечение между группой контрольных крыс и крыс подгруппы 1.2 (14 суток после трансплантации опухоли), что можно расценить как то, что 14 суток - малый срок для однозначной классификации различий между здоровыми и онкологическими образцами. Следует отметить, что достоверных отличий в содержании белка между подгруппами 1.1 и 1.2 не отмечено (табл. 1).

Из рис. 10 сложно оценить разделимость данных для здоровых крыс (подгруппа 2.1) и подгрупп 2.2 (14 суток после трансплантации опухоли) и 2.3 (28 суток после трансплантации опухоли). Все кластеры разрежены. Следует отметить, что животные группы 2 имели повышенный уровень глюкозы в сыворотке крови, а трансплантация опухоли привела к ее значительному росту. В то же время достоверных отличий по содержанию как общего белка, так и альбумина, в подгруппах 2.2 и 2.3 не наблюдается в отличие от животных группы 1 (табл. 1). Различия в разделении групп 1 и 2 в пространстве главных компонент связаны с различием в составе сыворотки крови.

Таким образом, использование метода главных компонент позволяет визуализировать спектры КРС сыворотки крови, определить значимые (по величине дисперсии) признаки. Визуальный анализ полученных рисунков позволяет выявить отличие спектров КРС сыворотки крови контрольных крыс от спектров сыворотки крови крыс на 28-е сутки после трансплантации опухоли для группы 1 и указывает на искажение результатов для группы 2 экспериментальных животных, которое может быть обусловлено влиянием повышенного уровня глюкозы. В то же время результаты, полученные для групп животных через 14 суток после трансплантации опухоли, свидетельствуют о том, что этот срок недостаточен для проведения полноценной классификации. 


\section{Заключение}

В настоящей работе проведено исследование спектров КРС сыворотки крови крыс в динамике развития экспериментального рака печени. Были исследованы образцы сыворотки крови здоровых крыс и крыс через 14 и 28 суток после трансплантации опухоли. Показано, что развитие экспериментального рака печени приводит к уменьшению концентрации как общего белка, так и альбумина. Наибольшие изменения в спектрах КРС наблюдаются в диапазоне $1200-1700 \mathrm{~cm}^{-1}$. Показано, что интенсивность полосы амид I отличается в спектрах сыворотки крови в зависимости от стадии развития холангиокарциномы и коррелирует с концентрацией белка в образцах. Для животных с повышенным уровнем глюкозы показано статистически значимое увеличение концентрации глюкозы по сравнению с животными с нормальным уровнем глюкозы, которое, возможно, приводит к гликированию белков и изменению формы полосы амид I. Появление полосы на $1670 \mathrm{~cm}^{-1}$, возможно, свидетельствует об увеличении содержания $\beta$ структурных элементов в конформации белка и наиболее ярко выражено в спектрах образцов группы 2.3, в которых опухоль развивалась 28 суток. Использование метода главных компонент позволяет разделять спектры КРС сыворотки крови контрольных крыс от спектров сыворотки крови крыс на 28 сутки после трансплантации опухоли. В то же время срок в 14 суток после трансплантации опухоли недостаточен для проведения полноценной классификации.

\section{Финансирование работы}

Работа выполнена при частичной финансовой поддержке грантов РФФИ 17-00-00275 (17-00-00270, 1700-00186 и 17-00-00272) и 19-52-55004 (при поддержке РФФИ и ГФЕН). Работа выполнена в рамках Программы фундаментальных научных исследований государственных академий наук на 2013-2020 годы, направление III.23.2.10.

\section{Конфликт интересов}

Авторы заявляют, что у них нет конфликта интересов.

\section{Список литературы}

[1] Daviss B. // The Scientist. 2005. V. 19. N 8. P. 25.

[2] Montanez J.E., Peters J.M., Correll J.B., Gonzalez F.J., Patterson A.D. // Toxicol Pathol. 2013. V. 41. N 2. P. 410. doi $10.1177 / 0192623312466960$

[3] Diem M., Mazur A., Lenau K., Schubert J., Bird B., Miljkovič M., Krafft C., Popp J. // J. Biophotonics. 2013. V. 6. N 11-12. P. 855. doi 10.1002/jbio.201300131

[4] Krafft C., Dietzek B., Schmitt M., Popp J. // J. Biomed. Opt. 2012. V. 17. N 4. P. 040801.

[5] Li Q.B., Hao C., Xu Z. // Sensors. 2017. V. 17. P. 627. doi $10.3390 / \mathrm{s} 17030627$
[6] Kong K., Kendall C., Stone N., Notingher I. // Adv. Drug Deliv. Rev. 2015. V. 89. P. 121.

[7] Qi D., Berger A.J. // Appl. Opt. 2007. V. 46. N 10. P. 1726.

[8] Pichardo-Molina J.L., Frausto-Reyes C, Barbosa-Garcia'O., Huerta-Franco R., González-Trujillo J.L., Ramírez-Alvarado C.A., Gutiérrez-Juárez G., Medina-Gutiérrez C. // Lasers. Med. Sci. 2007. V. 22. P. 229.

[9] Taleb I., Thiéfin G., Gobinet C., Untereiner V., BernardChabert B., Heurgué A., Truntzer C., Hillon P., Manfait M., Ducoroy P., Sockalingum G.D. // Analyst. 2013. V. 138. P. 4006. doi 10.1039/C3AN00245D

[10] Uckermann O., Yao W., Juratli T.A., Galli R., Leipnitz E., Meinhardt M., Koch E., Schackert G., Steiner G., Kirsch M. // J. Neurooncol. 2018. V. 139. N 2. P. 261. doi 10.1007/s11060018-2883-8

[11] Livermore J., Lau K., Scott C., Plaha P., Vallance C., Ansorge O. // Neuro Oncol. 2017. V. 19. Suppl 1. P. i14-i15.

[12] Kistenev Y.V., Borisov A.V., Shapovalov A.V. // Proc. SPIE. 2015. V. 9810. P. 9810-58. doi 10.1117/12.2225575

[13] Kistenev Y.V., Shapovalov A.V., Borisov A.V., Vrazhnov D.A., Nikolaev V.V., Nikiforova O.Y. // Proc. SPIE. 2015. V. 9810. P. 98101Y-1. doi 10.1117/12.2225565

[14] Kistenev Y.V., Borisov A.V., Kuzmin D.A., Penkova O.V., Kostyukova N.Y., Karapuzikov A.A. // J. Biomed. Opt. 2017. V. 22. N 1. P. 017002.

[15] Kistenev Y.V., Karapuzikov A.I., Kostyukova N.Y., Starikova M.K., Boyko A.A., Bukreeva E.B., Bulanova A.A., Kolker D.B., Kuzmin D.A., Zenov K.G., Karapuzikov A.A. // J. Biomed. Opt. 2015. V. 20. N 6. P. 065001.

[16] Pan Q.-X., Su Z.-J., Zhang J.-H., Wang C.-R., Ke S.-Y. // Mol. Clin. Oncol. 2017. V. 6. N 4. P. 566.

[17] Бучарская А.Б., Дихт Н.И., Афанасьева Г.А., Терентюк Г.С., Захарова Н.Б., Маслякова Г.Н., Хлебцов Б.Н., Хлебцов Н.Г. // Саратовский научно-медицинский журнал. 2015. T. 11. № 2. С. 107.

[18] Назаров М.М., Черкасова О.П., Лазарева Е.Н., Бучарская А.Б., Наволокин Н.А., Тучин В.В., Шкуринов А.П. // Опт. и спектр. 2019. Т. 126. № 6. С. 799.

[19] International Guiding Principles for Biomedical Research Involving Animals (2012) CIOMS \& ICLAS. http://www.cioms.ch/index.php/12-newsflash/227-ciomsand-iclas-release-the-newinternational-guiding-principles-forbiomedical-researchinvolving-animals

[20] Brandt N.N., Chikishev A.Yu., Chulichkov A.I., Ignatiev P.A., Lebedenko S.I., Voronina O.V. // Laser Phys. 2004. N 14 (11), P. 1386-1392.

[21] Brandt N.N., Brovko O.O., Chikishev A.Y., Paraschuk O.D. // Appl. Spectrosc. 2006. V. 60. N 3. P. 288.

[22] Baek S.-J. Park A., Ahn Y.-J., Choo J. // Analyst. 2015. V. 140. N 1. P. 250. doi 10.1039/c4an01061b

[23] Eilers P.H.C., Boelens H.F.M. // Leiden University Medical Centre Report. 2005. V. 1. N 1. P. 5. doi 10.1039/c4an01061b

[24] Электронный ресурс. Режим доступа: https://github.com/charlesll/rampy

[25] Фундаментальная и клиническая физиология: Учебник для студ. высш. учеб. заведений / Под. ред. Камкина А.Г., Каменского А.А. М.: Издательский центр „Академия“, 2004. $1072 \mathrm{c}$.

[26] Кручинин В.Н., Кручинина М.В., Прудникова Я.И., Спесивцев Е.В., Рыхлицкий С.В., Володин В.А., Шеховцов С.В., Пельтек С.Е. // Опт. и спектр. 2019. Т. 127. № 1. C. 170 . 
[27] Shamina L.A., Bratchenko I.A., Artemyev D.N., Myakinin O.O., Moryatov A.A., Orlov A.E., Kozlov S.V., Zakharov V.P. // Proc. SPIE. Saratov Fall Meeting 2017: Optical Technologies in Biophysics and Medicine XIX. 2018. V. 10716. P. 107160D. doi 10.1117/12.2315073

[28] Bratchenko L.A., Bratchenko I.A., Lykina A.A., Komarova M.V., Artemyev D.N., Myakinin O.O., Moryatov A.A., Davydkin I.L., Kozlov S.V., Zakharovet V.P. // J. Raman Spectrosc. 2019. P. 1. doi 10.1002/jrs.5762

[29] Rygula A., Majzner K., Marzec K.M., Kaczor A., Pilarczyk M., Baranska M. // J. Raman Spectrosc. 2013. V. 44. P. 1061.

[30] Guevara E., Torres-Galván J.C., Ramírez-Elías M.G., Luevano-Contreras C., González F.J. // Biomed. Opt. Express. 2018. V. 9. P. 4998.

[31] Neugebauer U., Clement J.H., Bocklitz T., Krafft C., Popp J. // J. Biophoton. 2010. V. 3. N 8-9. P. 579. doi 10.1002/jbio.201000020

[32] Shirshin E., Cherkasova O., Tikhonova T., Berlovskaya E., Priezzhev A., Fadeev V. // J. Biomed. Opt. 2015. V. 20. N 5. P. 51033. doi 10.1117/1.JBO.20.5.051033

[33] Vetter S.W. // Adv. Clin. Chem. 2015. V. 72. P. 205.

[34] Huang Y.-T., Liao H.-F., Wang S.-L., Lin S.-Y., Huang Y.-T. // AIMS Biophysics. 2016. V. 3. N 2. P. 247. doi 10.3934/biophy.2016.2.247

[35] Fagnano C., Fini G., Torreggiani A. // J. Molecular Struct. 1995. V. 348. P. 9.

[36] Marx J., Berjot M., Alix A.J.P. // J. Raman Spectrosc. 1987. V. 18. N 4. P. 289.

[37] Williams R.W. // Methods Enzymol. 1986. V. 130. P. 311.

[38] Maiti N.C., Apetri M.M., Zagorski M.G., Carey P.R., Anderson V.E. // J. Am. Chem. Soc. 2004. V. 126. N 8. P. 2399.

[39] Byler D.M., Susi H. // Biopolymers. 1986. V. 25. N 3. P. 469.

[40] Нурхаметов А.Х., Елякова Е.Г., Ефремов Е.С., Мирошников А.И. // Биоорганическая химия. 1981. Т. 7. № 1. С. 16.

[41] Carey P.R. Biochemical Applications of Raman and Resonance Raman Spectroscopies. New York: Academic Press, 1982.

[42] Roessl U., Leitgeb S., Pieters S., De Beer T., Nidetzky B. // J. Pharm Sci. 2014. V. 103. N 8. P. 2287.

[43] Herrero A.M., Carmona P., Jimenez-Colmenero F., RuizCapillas C. // Handbook of vibrational spectroscopy / Ed. by Chalmers J.M., Griffiths P.R., Hoboken, New Jersey: JohnWiley \& Sons, 2010. P. 315-328.

[44] Yang H, Yang S, Kong J., Dong A., Yu S. // Nat. Protoc. 2015. V. 10. N 3. P. 382. doi 10.1038/nprot.2015.024.

[45] Dingari N.C., Horowitz G.L., Kang J.W., Dasari R.R., Barman I. // PLoS ONE. 2012. V. 7. N 2. P. e32406.

[46] Ishigaki M., Hashimoto K., Sato H., Ozaki Y. // Scientific Rep. 2017. V. 7. P. 43942.

[47] Bogliolo L., Murrone O., Di Emidio G., Piccinini M., Ariu F., Ledda S., Tatone C. // J. of Assisted Reproduction and Genetics. 2013. V. 30. N 7. P. 877.

[48] Tarcea N., Popp J. // Raman Spectroscopy Applied to Earth Sciences and Cultural Heritage / Ed. by Dubessy J., Caumon M.-C., Rull F. The Mineralogical Society of Great Britain \& Ireland, 2012. V. 12. 